This article has been accepted for publication in Monthly Notices of the Royal Astronomical Society (C): 2018 The Authors. Published by Oxford University Press on behalf of the Royal Astronomical Society. All rights reserved. 


\title{
On the ISW-cluster cross-correlation in future surveys
}

\author{
Mario Ballardini ${ }^{\ominus}$, $, 2,3,4 \star$ Daniela Paoletti, ${ }^{3,4 \star}$ Fabio Finelli, ${ }^{3,4}$ Lauro Moscardini, $, 2,5,4$ \\ Barbara Sartoris ${ }^{6,7}$ and Luca Valenziano ${ }^{3,4}$ \\ ${ }^{1}$ Department of Physics and Astronomy, University of the Western Cape, Cape Town 7535, South Africa \\ ${ }^{2}$ Dipartimento di Fisica e Astronomia, Alma Mater Studiorum Università di Bologna, Via Gobetti, 93/2, I-40129 Bologna, Italy \\ ${ }^{3}$ INAF/OAS Bologna, via Gobetti 101, I-40129 Bologna, Italy \\ ${ }^{4}$ INFN, Sezione di Bologna, Via Berti Pichat 6/2, I-40127 Bologna, Italy \\ ${ }^{5}$ INAF/OAS Bologna, via Gobetti 93/3, I-40129 Bologna, Italy \\ ${ }^{6}$ Dipartimento di Fisica, Sezione di Astronomia, Università di Trieste, Via Tiepolo 11, I-34143 Trieste, Italy \\ ${ }^{7}$ INAF - Osservatorio Astronomico di Trieste, Via Tiepolo 11, I-34143 Trieste, Italy
}

Accepted 2018 October 11. Received 2018 October 1; in original form 2017 December 11

\begin{abstract}
We investigate the cosmological information contained in the cross-correlation between the integrated Sachs-Wolfe (ISW) of the cosmic microwave background (CMB) anisotropy pattern and galaxy clusters from future wide surveys. Future surveys will provide cluster catalogues with a number of objects comparable with galaxy catalogues currently used for the detection of the ISW signal by cross-correlation with the CMB anisotropy pattern. By computing the angular power spectra of clusters and the corresponding cross-correlation with $\mathrm{CMB}$, we perform a signal-to-noise ratio (SNR) analysis for the ISW detection as expected from the eROSITA and the Euclid space missions. We discuss the dependence of the SNR of the ISWcluster cross-correlation on the specifications of the catalogues and on the reference cosmology. We forecast that the SNRs for ISW-cluster cross-correlation are slightly smaller compared to those which can be obtained from future galaxy surveys but the signal is expected to be detected at high significance, i.e. more than $>3 \sigma$. We also forecast the joint constraints on parameters of model extensions of the concordance $\Lambda$ cold dark matter $(\Lambda \mathrm{CDM})$ cosmology by combining CMB and the ISW-cluster cross-correlation.
\end{abstract}

Key words: galaxies: clusters: general - cosmic background radiation-cosmological parameters.

\section{INTRODUCTION}

One of the key predictions of the recent accelerated expansion is the late integrated Sachs-Wolfe (ISW) effect (Sachs \& Wolfe 1967) in the cosmic microwave background (CMB) anisotropy pattern. The ISW effect is caused by the time evolution of the gravitational potentials encountered by CMB photons during their journey from the last scattering surface to the observer. The change of the gravitational potentials on large scales is caused by the accelerated expansion of the Universe driven by dark energy (Kofman \& Starobinsky 1985) and is therefore correlated with density fluctuations at low redshifts.

The late ISW effect is just a small fraction of the CMB temperature anisotropy signal for a $\Lambda$ cold dark matter $(\Lambda \mathrm{CDM})$ concordance cosmology and is maximum at largest angular scales (Kofman \& Starobinsky 1985), where cosmic variance is dominant. An extraction of the ISW effect was predicted as feasible by cross-

^E-mail: ballardini@iasfbo.inaf.it (MB); daniela.paoletti@inaf.it (DP) correlating CMB with large-scale structure (LSS) tracers of the matter distribution (Crittenden \& Turok 1996). Several measurements of the ISW-LSS cross-correlation have been reported since the release of the WMAP first-year data (Nolta et al. 2004) by using different dark matter (DM) tracers. These measurements have been performed by using different estimators in different domains (e.g. Afshordi, Loh \& Strauss 2004; Boughn \& Crittenden 2004; Fosalba \& Gaztanaga 2004; Vielva, Martinez-Gonzalez \& Tucci 2006; McEwen et al. 2007; Giannantonio et al. 2008; Ho et al. 2008; Hernandez-Monteagudo 2010; Schiavon et al. 2012; Bianchini, Renzi \& Marinucci 2016; Stölzner et al. 2017). See Dupe et al. (2011) and references therein for a review of pre-Planck ISW measurements.

A detection at $2.9 \sigma$ level of the ISW effect has beeen obtained by Planck Collaboration XXI (2016) by cross-correlating the Planck temperature map with a compilation of publicly available galaxy surveys (see Planck Collaboration XIX 2014 for the results based on the nominal mission). This cross-correlation measurement of the ISW effect is compatible with the predictions of a concordance $\Lambda$ CDM model with $\Omega_{\Lambda} \sim 0.7$ (Planck Collaboration XXI 2016). 
Cross-correlation between CMB lensing and matter tracers improve the significance of the ISW detection (see Manzotti \& Dodelson 2014; Ferraro, Sherwin \& Spergel 2015; Planck Collaboration XXI 2016; Shajib \& Wright 2016). Note also that the ISW could be targeted by using its cross-correlation with CIB (Ilic et al. 2011) or tSZ (Taburet et al. 2011).

Future LSS experiments, such as DESI ${ }^{1}$ (Levi et al. 2013; Aghamousa et al. 2016a,b), Euclid ${ }^{2}$ (Laureijs et al. 2011; Amendola et al. 2013), $\operatorname{LSST}^{3}$ (Abell et al. 2009), and SKA ${ }^{4}$ (Maartens et al. 2015), will provide surveys with a larger number of objects and sky fraction probing a larger volume, then allowing a better determination of the ISW signal. The next generation of LSS surveys will not only lead to an improvement in the ISW measurement with matter tracers already employed for this purpose but could also provide different catalogues of matter tracers suitable for novel detections of the ISW effect (Raccanelli et al. 2015, 2016; Pourtsidou, Bacon \& Crittenden 2017).

Current cluster surveys are not optimal for the detection of the ISW effect since photometric catalogues are limited to small redshift $z \lesssim 0.4$ (Rozo et al. 2010) and X-ray catalogues cover small patches of the sky (Mantz et al. 2015). ${ }^{5}$

Otherwise, the cluster catalogues from the next generation of LSS surveys are indeed expected to contain a higher number of clusters over a wider redshift range. These future catalogues will allow precision cosmology with clusters, as from the cosmological parameter forecasts from the Euclid cluster survey (Sartoris et al. 2016). It is therefore interesting to study the potential for an ISW detection by a cross-correlation with future cluster catalogues as matter tracers.

In this paper, by computing the angular power spectra of cluster counts and of their cross-correlation with $\mathrm{CMB}$, we perform a signal-to-noise ratio (SNR) analysis for the ISW detection with two representative examples for future cluster catalogues, as those expected from the eROSITA (Predehl et al. 2010) and Euclid (Laureijs et al. 2011) space missions and we forecast how ISW-cluster crosscorrelation could improve the constraints on cosmological parameters for different extended models. This analysis is complementary to the stacking of CMB data in correspondence of superclusters (Granett et al. 2008; Papai, Szapudi \& Granett 2011; Ilic et al. 2011; Planck Collaboration XIX 2014; Planck Collaboration XXI 2016), whose theoretical interpretation is more difficult, although consistent with $\Lambda \mathrm{CDM}$.

Our paper is organized as follows. In Section 2, we review the angular power spectrum of the cross-correlation between CMB and a generic matter tracer, such as clusters or galaxies. In Section 3, we review the calculations of the bias and the number density distribution for cluster catalogues. In Section 4, we describe the future surveys considered in our work: eROSITA and Euclid for clusters, Euclid photometric and spectroscopic surveys for galaxies. In Section 5, we compute the auto- and CMB cross-correlation angular power spectra for these surveys and perform an SNR analysis. In Section 6, we present forecasted constraints on cosmological parameters of extensions of the concordance $\Lambda$ CDM model by com-

\footnotetext{
${ }^{1}$ http://desi.lbl.gov/

${ }^{2} \mathrm{http}: / /$ sci.esa.int/euclid/

${ }^{3} \mathrm{http}: / /$ www.lsst.org/

${ }^{4}$ http://www.skatelescope.org/

${ }^{5}$ The only analysis of the late ISW using clusters has been performed by Granett, Neyrinck \& Szapudi (2008) stacking WMAP data behind 50 superclusters (and 50 supervoids) to study the residual signal from the detection of the thermal SZ effect.
}

bining CMB and the CMB-LSS cross-correlation. We draw our conclusions in Section 7.

Throughout this work, we adopt a fiducial cosmological model compatible with the most recent Planck data (Aghanim et al. 2016), corresponding to $\omega_{\mathrm{b}} \equiv \Omega_{\mathrm{b}} h^{2}=0.02214, \omega_{\mathrm{c}} \equiv \Omega_{\mathrm{c}} h^{2}=0.1206$, $H_{0}=66.89, \tau=0.0581, n_{\mathrm{s}}=0.9625$, and $\log \left(10^{10} A_{\mathrm{s}}\right)=3.053$.

\section{CROSS-CORRELATION OF ISW EFFECT WITH NUMBER COUNTS}

We consider the projected density contrast of a tracer of matter X (clusters or galaxies in this paper) in the direction $\hat{n}$ as

$\delta_{\mathrm{X}}(\hat{n})=\int \mathrm{d} z b_{\mathrm{X}}(z) \frac{\mathrm{d} N_{\mathrm{X}}}{\mathrm{d} z} \delta_{\mathrm{m}}(\hat{n}, z)$.

In equation (1), we denote by $b_{\mathrm{X}}(z)$ the linear bias and by $\mathrm{d} N_{\mathrm{X}} / \mathrm{d} z$ the redshift distribution of the tracer. The observed tracer density is correlated with the ISW contribution to temperature fluctuations in direction $\hat{m}$ :

$\frac{\delta T_{\mathrm{ISW}}}{T}(\hat{m})=-\int \mathrm{d} z e^{-\tau(z)}\left(\frac{\mathrm{d} \Phi}{\mathrm{d} z}(\hat{m}, z)+\frac{\mathrm{d} \Psi}{\mathrm{d} z}(\hat{m}, z)\right)$,

where $\Phi$ and $\Psi$ are the gravitational potentials in the longitudinal gauge and $e^{-\tau(z)}$ is the visibility function.

With these assumptions, the angular power spectrum of the tracer $C_{\ell}^{\mathrm{XX}}$ and of its cross-correlation with $\mathrm{CMB}$ temperature $C_{\ell}^{\mathrm{TX}}$ are respectively

$$
\begin{aligned}
C_{\ell}^{\mathrm{XX}} & =4 \pi \int \frac{\mathrm{d} k}{k} \Delta^{2}(k)\left[I_{\ell}^{\mathrm{X}}(k)\right]^{2}, \\
C_{\ell}^{\mathrm{TX}} & =4 \pi \int \frac{\mathrm{d} k}{k} \Delta^{2}(k) I_{\ell}^{\mathrm{ISW}}(k) I_{\ell}^{\mathrm{X}}(k),
\end{aligned}
$$

where we denote by $\Delta^{2}(k)$ the scale invariant matter power spectrum $\Delta^{2}(k) \equiv k^{3} P(k) /\left(4 \pi^{2}\right)$, and define the kernels of the counts distribution and of the cross-correlation as

$$
\begin{aligned}
I_{\ell}^{\mathrm{X}}(k) & =\int \mathrm{d} z b_{\mathrm{X}}(z) \frac{\mathrm{d} N_{\mathrm{X}}}{\mathrm{d} z} D(z) j_{\ell}(k \chi(z)), \\
I_{\ell}^{\mathrm{ISW}}(k) & =-\int \mathrm{d} z e^{-\tau}\left(\frac{\mathrm{d} \Phi}{\mathrm{d} z}+\frac{\mathrm{d} \Psi}{\mathrm{d} z}\right) j_{\ell}(k \chi(z)),
\end{aligned}
$$

where $D(z)$ is the growth factor and $\chi(z)$ is the conformal distance.

\section{CLUSTER NUMBER DENSITY}

We now review the computation of the number density for clusters and the corresponding bias that we will use in equation (3). The number density of expected clusters within the solid angle $\Delta \Omega$ is

$\frac{\mathrm{d} N(z)}{\mathrm{d} z}=\int_{\Delta \Omega} \mathrm{d} \Omega \frac{\mathrm{d} V_{\mathrm{c}}}{\mathrm{d} z \mathrm{~d} \Omega} \int_{0}^{\infty} \mathrm{d} M X(M, z) n_{\mathrm{h}}(M, z)$,

where $V_{\mathrm{c}}$ is the comoving volume, $n_{\mathrm{h}}(M, z)$ and $X(M, z)$ are the DM halo mass function and the survey characteristic function, respectively.

\subsection{Halo mass function}

The distribution $n_{\mathrm{h}}\left(M_{\mathrm{h}}, z\right)$ of DM haloes in mass, position, and redshift describes the overall abundance and clustering properties of galaxies, and the dependence on the underlying cosmological 
model. As halo mass function, we use (Tinker et al. 2008)

$$
\begin{aligned}
n_{\mathrm{h}}(M, z) & =-f(\sigma) \frac{\rho_{\mathrm{m}}}{M} \frac{\mathrm{d} \ln \sigma(M, z)}{\mathrm{d} M}, \\
f(\sigma) & =C_{1}(z)\left[1+\left(\frac{\sigma(M, z)}{C_{3}(z)}\right)^{-C_{2}(z)}\right] \mathrm{e}^{-C_{0} / \sigma(M, z)^{2}},
\end{aligned}
$$

where $\rho_{\mathrm{m}}$ is the total physical matter density and $\sigma(M, z)$ is the root mean square density fluctuation within a sphere. The variance of the fractional density fluctuation,

$\sigma^{2}(M, z)=\int_{0}^{\infty} \frac{k^{2} \mathrm{~d} k}{2 \pi^{2}} P(k, z) W^{2}(k, M)$,

is calculated by integrating the linear matter power spectrum $P(k, z)$ smoothed by a top-hat window in real space window function $W(k$, $M)$.

According to Tinker et al. (2008), we parametrize the redshift evolution of the parameters of the mass function in equation (8) as different power-law functions of $(1+z)$

$$
\begin{aligned}
C_{1}(z) & =C_{1,0}(1+z)^{-0.14}, \\
C_{2}(z) & =C_{2,0}(1+z)^{-0.06}, \\
C_{2}(z) & =C_{3,0}(1+z)^{-\gamma}, \\
\log (\gamma) & =-\left(\frac{0.75}{\log \left(\Delta_{c} / 75\right)}\right)^{1.2},
\end{aligned}
$$

where the subscript 0 denotes the value of the quantity at $z=0$ and $\Delta_{\mathrm{c}}$ defines the mean overdensity of the spherical haloes in terms of the critical density of the universe. The quantities $C_{0}, C_{1,0}, C_{2,0}$, $C_{3,0}$ depend on $\Delta_{\mathrm{c}}$ and we refer the reader to Tinker et al. (2008) for their numerical values. We use for our forecasts $\Delta_{c}=200$ and 500 for Euclid and eROSITA, respectively.

\subsection{Halo bias function}

We now specify the halo bias function, $b_{\mathrm{eff}}(z)$. The linear bias weighted by the halo mass function is

$b_{\mathrm{eff}}(z)=\frac{1}{n_{\mathrm{h}}(z)} \int_{0}^{\infty} \mathrm{d} M n_{\mathrm{h}}(M, z) b_{\mathrm{h}}(M, z) X(M, z)$,

where the integrated halo redshift distribution is

$n_{\mathrm{h}}(z)=\int_{0}^{\infty} \mathrm{d} M n_{\mathrm{h}}(M, z) X(M, z)$.

According to Tinker et al. (2010), the Eulerian halo bias is

$b_{\mathrm{h}}\left(M_{\mathrm{h}}, z\right)=1-c_{1} \frac{v^{c_{4}}}{v^{c_{4}}+\delta_{\mathrm{c}}^{c_{4}}}+c_{2} v^{c_{5}}+c_{3} v^{c_{6}}$,

where $v=\delta_{\mathrm{c}} / \sigma\left(M_{\mathrm{h}}, z\right)$ and $\delta_{\mathrm{c}}$ is the critical density for collapse (in all calculations we use $\delta_{\mathrm{c}}=1.686$ ) and the parameters of the halo bias function in equation (14) as a function of $\Delta_{c}$ are

$c_{1}=1+0.24 x \mathrm{e}^{-(4 / x)^{4}}$,

$c_{2}=0.183$,

$c_{3}=0.019+0.107 x+0.19 \mathrm{e}^{-(4 / x)^{4}}$,

$c_{4}=0.44 x-0.88$,

$c_{5}=1.5$,

$c_{6}=2.4$,

where $x \equiv \log \Delta_{\mathrm{c}}$.

\subsection{Survey characteristic function}

We express the survey characteristic completeness function in equation (7) as

$X(M, z)=\int_{M_{\lim }(z)}^{\infty} \mathrm{d} M^{\text {obs }} P\left(M^{\text {obs }} \mid M\right)$,

where $M_{\text {lim }}(z)$ represents the minimum value of the observed mass for a cluster to be included in the survey, and it is determined by the survey selection function. The function $P\left(M^{\text {obs }} \mid M\right)$ gives the probability that a cluster of true mass $M$ has a measured mass $M^{\text {obs }}$ and takes into account the uncertainties that a scaling relation introduces in the knowledge of the cluster mass. Following Lima \& $\mathrm{Hu}$ (2005) and Sartoris et al. (2010), the probability of assigning to a cluster of true mass $M$ an observed mass $M^{\text {obs }}$ can be well approximated with a lognormal density distribution:

$$
\begin{aligned}
P\left(M^{\mathrm{obs}} \mid M\right)= & \frac{1}{M^{\mathrm{obs}} \sqrt{2 \pi \sigma_{\log M}^{2}}} \\
& \times \exp \left[-\frac{\left(\ln M^{\mathrm{obs}}-B_{M}-\ln M\right)^{2}}{2 \sigma_{\log M}^{2}}\right],
\end{aligned}
$$

where the parameter $B_{\mathrm{M}}=B_{\mathrm{M}, 0}+\alpha \log (1+z)$ represents the fractional value of the systematic bias in the mass estimate and $\sigma_{\log M}^{2}=\sigma_{\log M, 0}^{2}-1+(1+z)^{2 \beta}$ is the intrinsic scattering. We treat $B_{\mathrm{M}, 0}=0, \alpha=0, \sigma_{\log M, 0}=0.2$, and $\beta=0.125$ as fiducial as in Sartoris et al. (2016) and we consider them as nuisance parameters in the following analysis.

\section{FUTURE SURVEYS}

In this section, we describe two future cluster catalogues suitable for the ISW detection by cross-correlation with CMB, i.e. those expected from eROSITA (Predehl et al. 2010) and Euclid (Laureijs et al. 2011). For comparison, we also present the specifications for the photometric and spectroscopic galaxy surveys expected from Euclid, which are expected to improve on the current statistical significance of the ISW detection with galaxy surveys.

\section{1 eROSITA cluster survey}

eROSITA ${ }^{6}$ (Predehl et al. 2010) is the primary science instrument onboard the Spectrum Roentgen-Gamma (SRG) satellite, ${ }^{7}$ launched in 2017.

eROSITA is expected to perform an X-ray all-sky survey with a sensitivity $\sim 30$ times better than ROSAT and to cover a redshift range of $0 \leq z \leq 1.5$ with a sky coverage of $27000 \mathrm{deg}^{2}$. We consider the $e$ ROSITA cluster selection function presented in Pillepich, Porciani \& Reiprich (2012), computed for a mass at $\Delta_{\mathrm{c}}=500$ with an exposure time of $1.6 \times 10^{3} \mathrm{~s}$ and a detection threshold of 50 photons. In addition, we adopt as lower cut for the minimum mass $5 \times 10^{13} \mathrm{M}_{\odot} h^{-1}$.

The resulting specifications of the eROSITA cluster catalogue (hereafter referred eR_cl) are summarized in Table 1, leading to a total number of clusters $\sim 8.1 \times 10^{4}$. The corresponding redshift density distribution and the linear bias for eROSITA are shown in Fig. 1.

\footnotetext{
${ }^{6}$ Extended ROentgen Survey with an Imaging Telescope Array, http://ww w.mpe.mpg.de/erosita/.

${ }^{7}$ http://hea.iki.rssi.ru/SRG/en/index.php
} 
Table 1. Summary of the characteristics of the surveys studied in this paper: fraction of the sky available $f_{\text {sky }}$ and surface density of tracer $\bar{n}_{\mathrm{X}}$.

\begin{tabular}{lcc}
\hline & $f_{\text {sky }}\left(\mathrm{deg}^{2}\right)$ & $\bar{n}_{\mathrm{X}}\left(\mathrm{deg}^{-2}\right)$ \\
\hline eR_cl & 27000 & 2.9 \\
Eu_cl_opt & 15000 & 72 \\
Eu_cl_con & 15000 & 10.8 \\
Eu_gal_sp & 15000 & 3960 \\
Eu_gal_ph & 15000 & 108000 \\
\hline
\end{tabular}
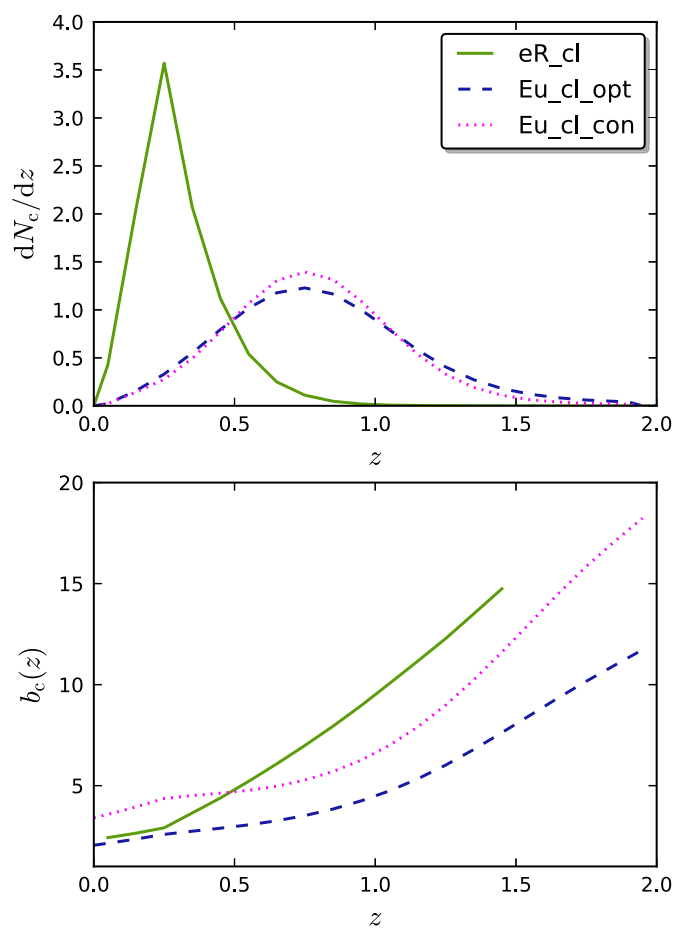

Figure 1. In the top panel, we plot the normalized cluster redshift distributions adopted for each survey: eROSITA (solid green), optimistic Euclid (dashed blue), and conservative Euclid (dotted magenta). In the bottom panel, we plot the corresponding linear bias.

\subsection{Euclid cluster survey}

The European Space Agency (ESA) Cosmic Vision mission Euclid (Laureijs et al. 2011) is scheduled to be launched in 2021, with the goal of exploring the dark sector of the Universe.

As selection function for the expected Euclid cluster catalogue we consider the one presented in Sartoris et al. (2016) computed for a mass at $\Delta_{c}=200$ with a threshold for the significance of the clusters detection of 3 in terms of the ratio between the cluster galaxy number counts and the field rms (hereafter referred Eu_cl_opt) with a sky coverage of $15000 \mathrm{deg}^{2}$ over a redshift range of $0.2 \leq z \leq 2$. We also consider a detection threshold of 5 as a more conservative case (hereafter referred Eu_cl_con). This selection function was obtained following a phenomenological approach. In summary, the number of cluster galaxies is obtained by integration of observed cluster luminosity function down to the $H$-band magnitude limit of the Euclid survey, and the variance in the field counts takes into account both Poisson noise and cosmic variance (see Sartoris et al. (2016) for more details). Even if the galaxy cluster mass selection
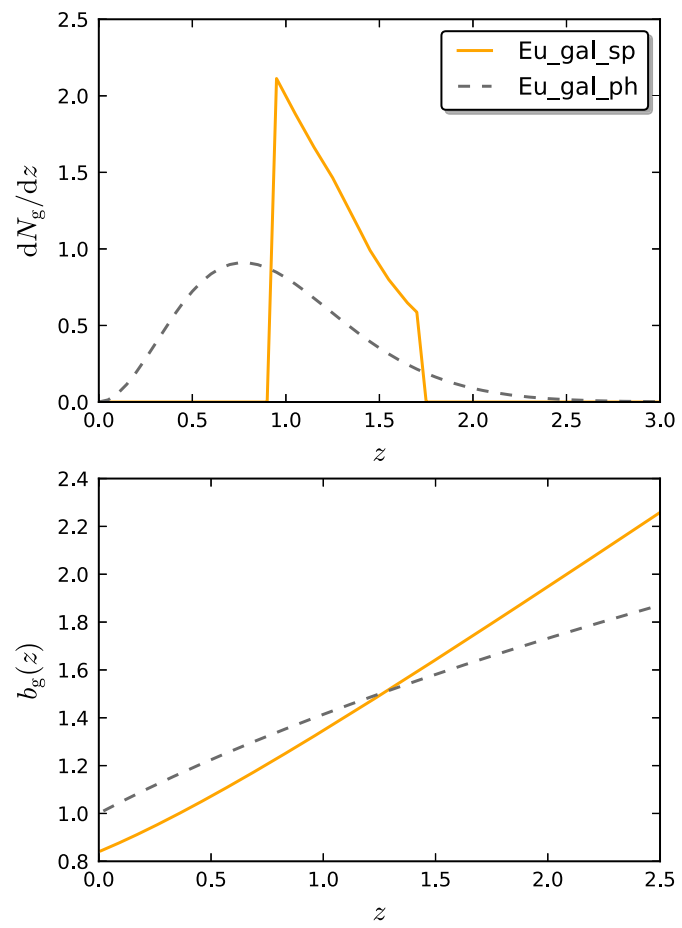

Figure 2. In the top panel, we plot the normalized galaxy redshift distribution adopted for each survey: spectroscopic Euclid (solid orange) and photometric Euclid (dashed grey). In the bottom panel, we plot the corresponding linear bias.

function used for the Euclid photometric survey is not constant (see Sartoris et al. 2016), a mass limit of $8 \times 10^{13} \mathrm{M}_{\odot} h^{-1}$ and $3 \times 10^{14} \mathrm{M}_{\odot} h^{-1}$ approximately mimics the counts of Eu_cl_opt and of Eu_cl_con, respectively.

The resulting specifications of the optimistic and conservative $E u$ clid expected cluster catalogues are summarized in Table 1, leading to a total number of clusters $1.3 \times 10^{6}$ and $2.0 \times 10^{5}$, respectively. See also Fig. 1 for the corresponding redshift density distribution and the linear bias.

\subsection{Euclid galaxy surveys}

Euclid will measure the galaxy clustering in a spectroscopic survey of tens of millions of $\mathrm{H} \alpha$ emitting galaxies and the cosmic shear in a photometric survey of billions of galaxies.

For the Euclid wide spectroscopic survey (hereafter referred Eu_gal_sp), we consider an area of $15000 \mathrm{deg}^{2}$. According to the updated predictions obtained by Pozzetti et al. (2016), the Euclid wide single-grism survey will reach a flux limit of $F_{\mathrm{H} \alpha}>$ $2 \times 10^{-16} \mathrm{erg} \mathrm{cm}^{-2} \mathrm{~s}^{-1}$ and will cover a redshift range of $0.9 \leq z$ $\leq 1.8$.

As specifications for the Euclid photometric survey (hereafter referred Eu_gal_ph), we adopt $\mathrm{d} N / \mathrm{d} z \propto z^{2} \exp \left[-\left(z / z_{0}\right)^{3 / 2}\right]$, with $z_{0}=z_{\text {mean }} / 1.412$ the peak of the distribution and $z_{\text {mean }}$ the median (Amendola et al. 2013). We choose $z_{\text {mean }}=0.9$, a surface density $\bar{n}_{\mathrm{g}}=30$ per $\operatorname{arcmin}^{2}$ and a bias $b_{\mathrm{g}}(z)=\sqrt{1+z}$ (Amendola et al. 2013).

The specifications for the Euclid spectroscopic and photometric galaxy surveys are summarized in Table 1. In Fig. 2, we plot the normalized redshift density distribution and the effective bias. 

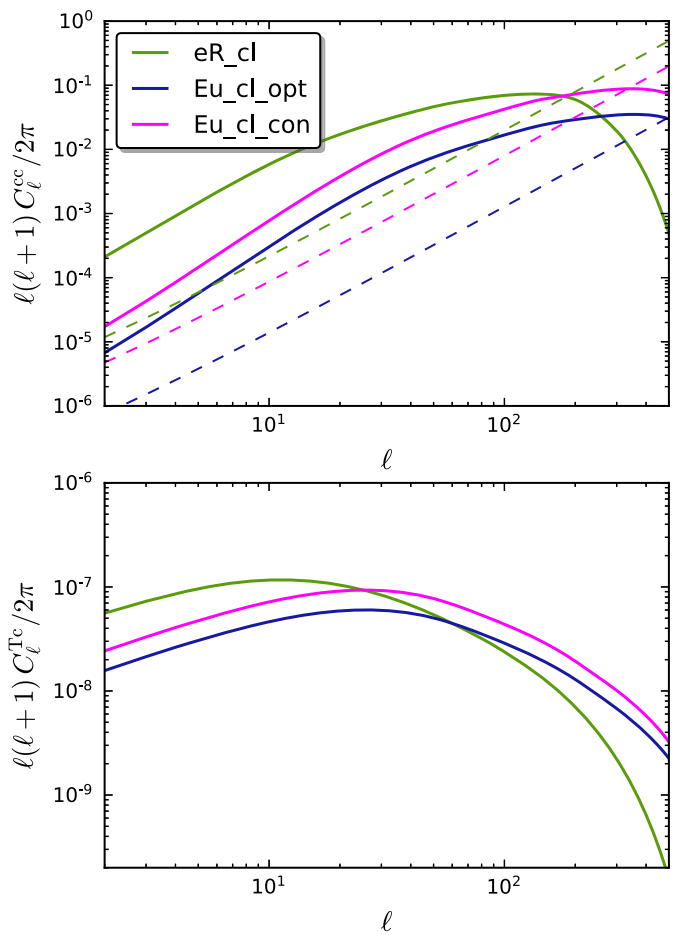

Figure 3. Cluster angular power spectra $C_{\ell}^{\mathrm{cc}}$ (top panel) and crosscorrelation angular power spectra $C_{\ell}^{\mathrm{Tc}}$ (bottom panel) for the three surveys of clusters: eROSITA (solid green), optimistic Euclid (solid blue), and conservative Euclid (solid magenta). In the top panel, the corresponding shot-noise (dashed lines) is also shown.

\section{AN SNR ANALYSIS}

We now investigate the detection level of the ISW effect by computing the SNR, which we define as (Cooray 2002; Afshordi 2004)

$$
\left(\frac{S}{N}\right)_{\mathrm{X}}^{2}=\sum_{\ell=2}^{\ell_{\max }}\left(\frac{S}{N}\right)_{\mathrm{X}, \ell}^{2},
$$

and

$$
\left(\frac{S}{N}\right)_{\mathrm{T}, \ell}^{2}=(2 \ell+1) \frac{f_{\text {sky }}^{\mathrm{X}}\left(C_{\ell}^{\mathrm{TX}}\right)^{2}}{\left(C_{\ell}^{\mathrm{TX}}\right)^{2}+\bar{C}_{\ell}^{\mathrm{TT}} \bar{C}_{\ell}^{\mathrm{XX}}},
$$

where $f_{\text {sky }}$ is the sky coverage of the survey and an overall bar stands for the sum of the signal and its noise. By $\bar{C}_{\ell}^{\mathrm{TT}}$ we denote the total CMB temperature angular power spectrum, which includes both the ISW and the Sachs-Wolfe terms, plus the effective noise:

$\bar{C}_{\ell}^{\mathrm{TT}}=C_{\ell}^{\mathrm{TT}}+\mathcal{N}_{\ell}^{\mathrm{T}}$.

At low and intermediate multipoles, Planck provides a measurement with a high SNR of the total anisotropy signal and the effective noise in temperature can therefore be neglected. The noise contribution for the mass tracer associated with a finite number of counts corresponds to a shot-noise as

$\mathcal{N}_{\ell}^{\mathrm{X}}=\frac{1}{\bar{n}_{\mathrm{X}}}$,

where $\bar{n}_{\mathrm{X}}$ is the surface density of tracers per steradian.

We show in Figs 3 and 4 the angular power spectra and the noises, for cluster and galaxy surveys respectively, calculated with the assumed fiducial cosmology and used in the SNR analysis.

The ISW effect leaves also an imprint on the E-mode polarization that can be quantified in analogy with equation (17), by substituting
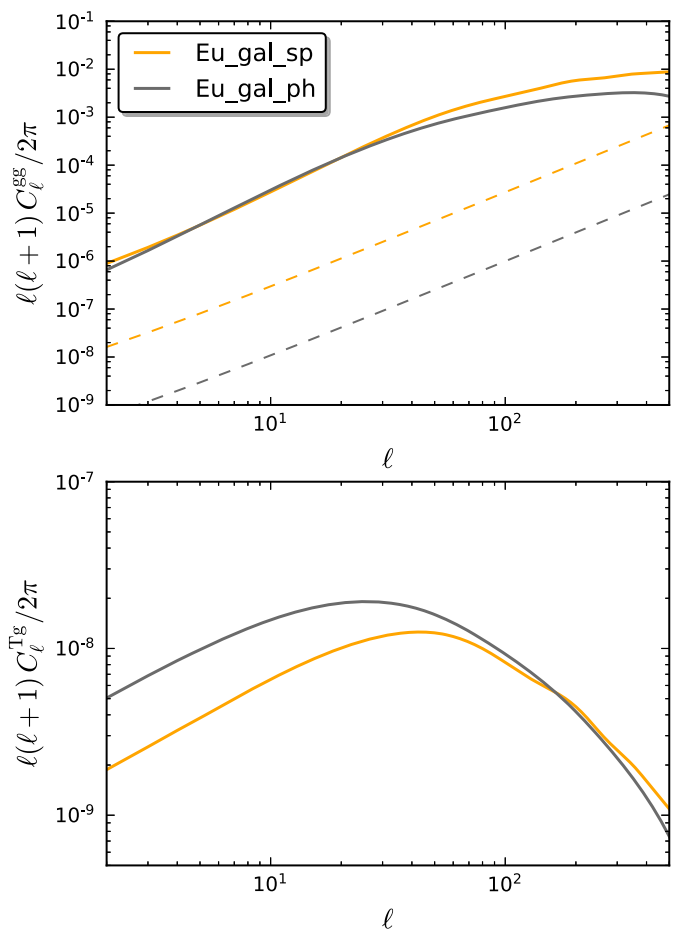

Figure 4. Galaxy angular power spectra $C_{\ell}^{\mathrm{gg}}$ (top panel) and crosscorrelation angular power spectra $C_{\ell}^{\mathrm{Tg}}$ (bottom panel) for two surveys of galaxies: spectroscopic Euclid (solid orange) and photometric Euclid (solid grey). In the top panel, the corresponding shot-noise (dashed lines) is also shown.

T with $\mathrm{E}$. We have checked that the SNR is at most 0.2 for the surveys considered here. Whereas the cross-correlation between the CMB E-mode polarization and LSS tracers is small as expected (Cooray \& Melchiorri 2006), the inclusion of polarization data allows a reduction of the error bars in the overall detection of the ISW effect (Frommert \& Enßlin 2009). Indeed, the E-mode polarization is correlated with primary temperature anisotropy (Zaldarriaga 1997) and therefore indirectly helps in disentangling the ISW effect. It is possible to extend the SNR analysis in equation (17) to the inclusion of polarization as in Frommert \& Enßlin (2009):

$$
\left(\frac{S}{N}\right)_{\mathrm{T}, \mathrm{E}, \ell}^{2}=(2 \ell+1) \frac{f_{\mathrm{sky}}^{\mathrm{X}}\left(C_{\ell}^{\mathrm{TX}}\right)^{2}}{\left(C_{\ell}^{\mathrm{TX}}\right)^{2}+\left(\bar{C}_{\ell}^{\mathrm{TT}}-\frac{\left(C_{\ell}^{\mathrm{TE}}\right)^{2}}{\bar{C}_{\ell}^{\mathrm{EE}}}\right) \bar{C}_{\ell}^{\mathrm{XX}}} .
$$

In the denominator of the latter equation, the power spectrum of temperature anisotropies is substituted by the corresponding one of the E-mode uncorrelated temperature map:

$a_{\ell \mathrm{m}}^{\mathrm{T}} \rightarrow a_{\ell \mathrm{m}}^{\mathrm{T}}-\frac{C_{\ell}^{\mathrm{TE}}}{\bar{C}_{\ell}^{\mathrm{EE}}} a_{\ell \mathrm{m}}^{\mathrm{E}}$.

The E-uncorrelated temperature map has been already introduced in the ISW-LSS cross-correlation analysis of the Planck 2015 release (Planck Collaboration XXI 2016).

We summarize in Table 2 the SNR for the cluster and galaxy catalogues introduced in Section 2 with the estimators in equations (17) and (21). Future cluster catalogues from eROSITA and Euclid could lead to a detection of the ISW effect at high significance $>3 \sigma$ thanks to the high number of clusters detected, i.e. a small noise level. We find that the SNR for these two cluster surveys will be comparable to the one obtainable from future galaxy surveys. In 
Table 2. SNR for ISW detection with future cluster and galaxy catalogues. The first row refers to the SNR with CMB temperature in equation (17); the second one to the estimate taking into account both CMB temperature and E-mode polarization as in equation (21).

\begin{tabular}{lccccc}
\hline & eR_cl & Eu_cl_opt & Eu_cl_con & Eu_gal_sp & Eu_gal_ph \\
\hline $\mathrm{T}$ & 2.3 & 3.5 & 3.3 & 2.2 & 3.7 \\
$\mathrm{~T}, \mathrm{E}$ & 2.8 & 4.1 & 3.9 & 2.5 & 4.3 \\
\hline
\end{tabular}

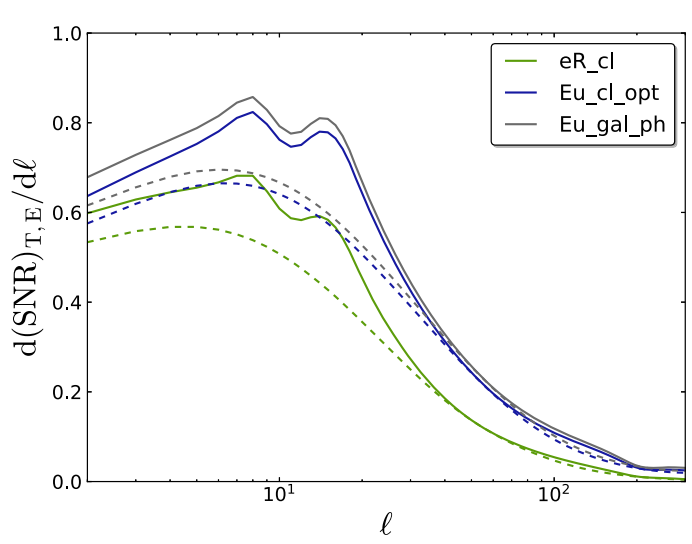

Figure 5. Comparison of $(S / N)_{\mathrm{X}, \ell}$ for temperature only (dashed) and including polarization (solid) according to equations (17) and (21), respectively, for the eROSITA and optimistic Euclid survey of clusters, together with the Euclid photometric survey of galaxies.

particular, for a Euclid survey, the SNR from clusters is smaller than the one expected from the photometric survey, but it is still larger than the SNR forecasted for the spectroscopic survey. The reason resides in the high bias expected for clusters that compensate the larger shot-noise in the observed cluster maps. The inclusion of CMB polarization information in the analysis increases the SNR by approximately 18 per cent as compared to the temperature only, with a weak dependence of the type of survey considered; this is fully consistent with Frommert \& Enßlin (2009); Giannantonio et al. (2012). We find the possibility of detecting the ISW effect at $4 \sigma$ both the cluster and the galaxy Euclid photometric surveys by adding the $\mathrm{CMB}$ polarization information. A comparison between the two SNR calculated from the two estimators is also shown in Fig. 5.

\subsection{Robustness from the specifications of the survey and cosmology}

We now test the dependence of the SNR obtainable for clusters on some of the specifications of the surveys, namely the smallest multipoles $\ell_{\text {min }}$, the covered sky fraction $f_{\text {sky }}^{\mathrm{X}}$, the maximum redshift $z_{\max }$, and the minimum detectable mass $M_{\min }$; for the sake of brevity, we present this analysis for the optimistic Euclid cluster (Eu_cl_opt) survey only and considering the inclusion of polarization in the SNR estimation.

Given the possibility to lose the first multipoles because of some mask effects and possible systematics on the largest scales, we show in Fig. 6 (top left-hand panel) the dependence of SNR on $\ell_{\min }$. The SNR remains approximately constant when removing the first few multipoles and is decreased approximately by $0.5 \sigma$ cutting the first 10 multipoles.

Bottom left-hand panel of Fig. 6 shows the SNR as a function of the observed sky fraction, which does not affect just equation (21) as $\mathrm{SNR} \propto \sqrt{f_{\text {sky }}^{\mathrm{X}}}$, but it changes also the amount of total clusters observed, i.e. the shot-noise error.

The SNR is sufficiently robust to a reduction of $z_{\max }$; the forecast SNR remains above a $4 \sigma$ detection for $z_{\max } \gtrsim 1.4$ for the optimistic Euclid cluster survey when the CMB polarization is added.

We finally study the dependence of the SNR when changing $M_{\min }$. We vertically shift a redshift independent selection function around $8 \times 10^{13} \mathrm{M}_{\odot}$, as representative of the selection function from (Sartoris et al. 2016) for the photometric Euclid cluster survey. The results in Fig. 6 (bottom left-hand panel) show that the expected selection function for Euclid would be optimal in terms of SNR.

We can conclude that for the ISW detection is more important to have wide surveys, covering a large fraction of the sky, than deeper ones, since the surveys analysed are already signal dominated on the scales relevant for the ISW.

We now briefly discuss the dependence of the SNR on the underlying cosmology. The cross-correlation power spectrum is sensitive to the total matter density $\Omega_{\mathrm{m}}$ and to the dark energy equation of state $w_{0}$. They both change the amplitude of $C_{\ell}^{\mathrm{TX}}$ (since for a flat universe an increased amount of matter density corresponds to have a smaller amount of dark energy density). Moreover, the peak of $C_{\ell}^{\mathrm{TX}}$ is shifted according to a different matter-dark energy equivalence. We show the dependence of the SNR on these two parameters in Fig. 7 by keeping fixed all the other cosmological parameters (note that we change $\sigma_{8}$ according to the variation of $\Omega_{\mathrm{m}}$ and $w_{0}$ ). The SNR varies less than $0.2 \sigma$ when we change $\Omega_{\mathrm{m}}$ up to $3 \sigma$ away from the Planck best fit (Aghanim et al. 2016). We find a stronger effect on the dark energy parameter of state $w_{0}$. Even a small shift of $\Delta w_{0}$ $\simeq 0.2$ would lead to a difference larger than $0.5 \sigma$ on the SNR. In particular, by assuming a fiducial cosmology with a smaller value of $w_{0}$ leads to a higher SNR.

\subsection{Combining clusters with galaxies}

Different LSS surveys can be combined to increase the significance of the ISW detection. The best improvement comes for uncorrelated LSS surveys for which the SNR squared can be simply added. In this case, we would obtain for the ISW detection an SNR of 3.2/4.1/4.0 for the combination of Eu_gal_sp and 4.3/5.1/5.0 for the combination of Eu_gal_ph with eR_cl, Eu_cl_opt, Eu_cl_con, respectively. However, this ideal case is usually reached for surveys that cover different redshifts or for distributions of tracers which peak at different redshifts.

The Euclid's cluster catalogue will be extracted from the photometric galaxy survey. Thus, they will trace by construction the same cosmological information mostly, having a similar redshift distribution and covering the same redshift range.

In order to take into account the cross-correlation between different LSS tracers, we extend the expression for the SNR (17) to

$$
\left(\frac{S}{N}\right)_{\mathrm{T}, \mathrm{c} \times \mathrm{g}, \ell}^{2}=\sum_{\mathrm{X}, \mathrm{Y}=\mathrm{c}, \mathrm{g}} C_{\ell}^{\mathrm{TX}}\left[\operatorname{Cov}_{\ell}^{-1}\right]_{\mathrm{XY}}, C_{\ell}^{\mathrm{TY}}
$$

where

$$
\left[\operatorname{Cov}_{\ell}\right]_{\mathrm{XY}}=\frac{C_{\ell}^{\mathrm{TX}} C_{\ell}^{\mathrm{TY}}+\bar{C}_{\ell}^{\mathrm{TT}} \bar{C}_{\ell}^{\mathrm{XY}}}{(2 \ell+1) \sqrt{f_{\text {sky }}^{\mathrm{X}} f_{\text {sky }}^{\mathrm{Y}}}} .
$$

In this case, the SNR by adding the information from Eu_gal_ph becomes 3.7 for both Eu_cl_opt and Eu_cl_con. There is no actual improvement compared to using Eu_gal_ph alone, but nevertheless 

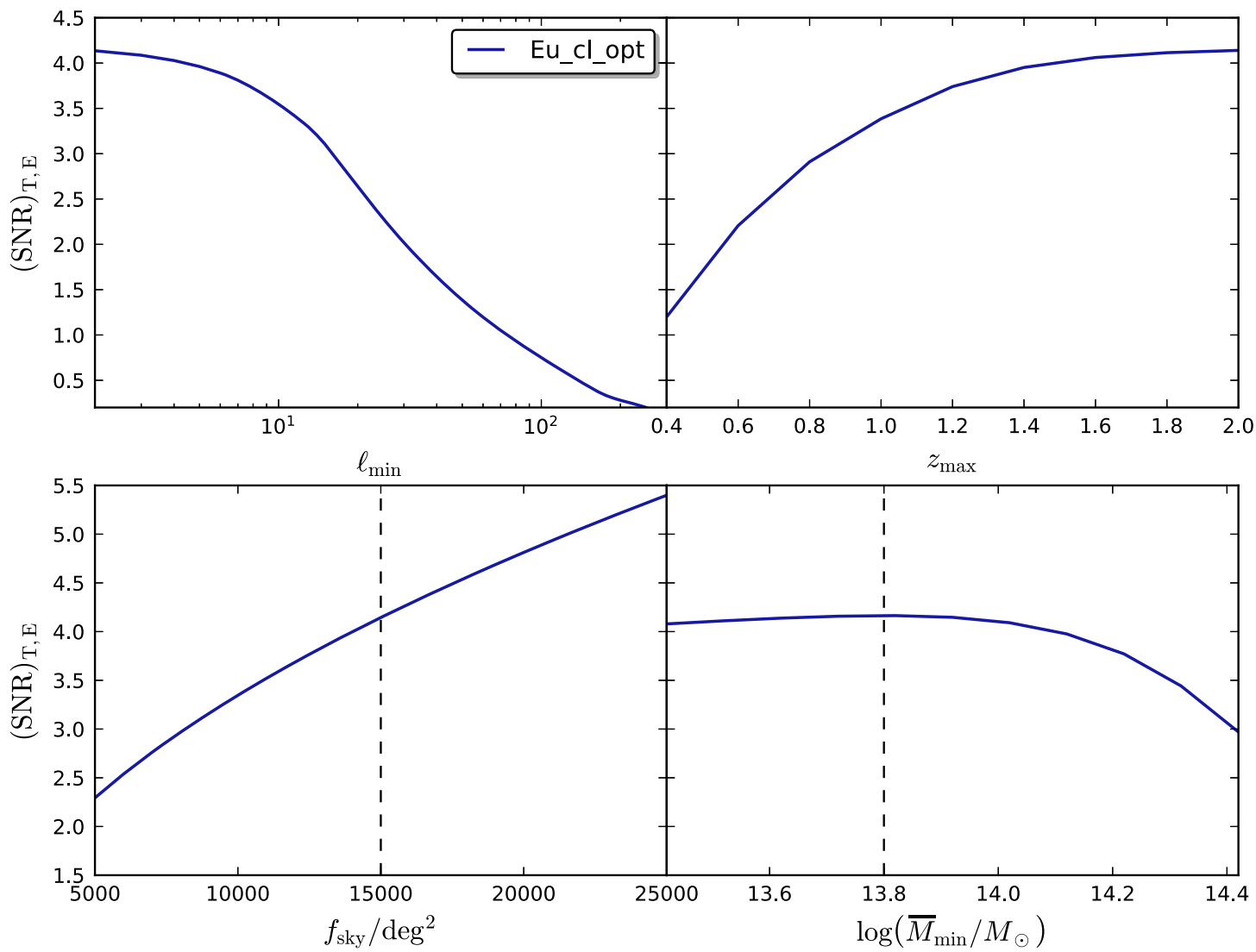

Figure 6. We plot the SNR for the optimistic Euclid cluster survey (Eu_cl_opt) as a function of $\ell_{\min }$ (top left), the maximum redshift probed by the survey (top right), the area of the sky survey (bottom left), and the minimum observed mass as over the redshift range $0.2 \leq z \leq 2$ (bottom right). Dashed line represents the expected SNR for the Eu_cl_opt specifications. All the SNR take into account of the contribution from CMB E-mode polarization according to equation (21).
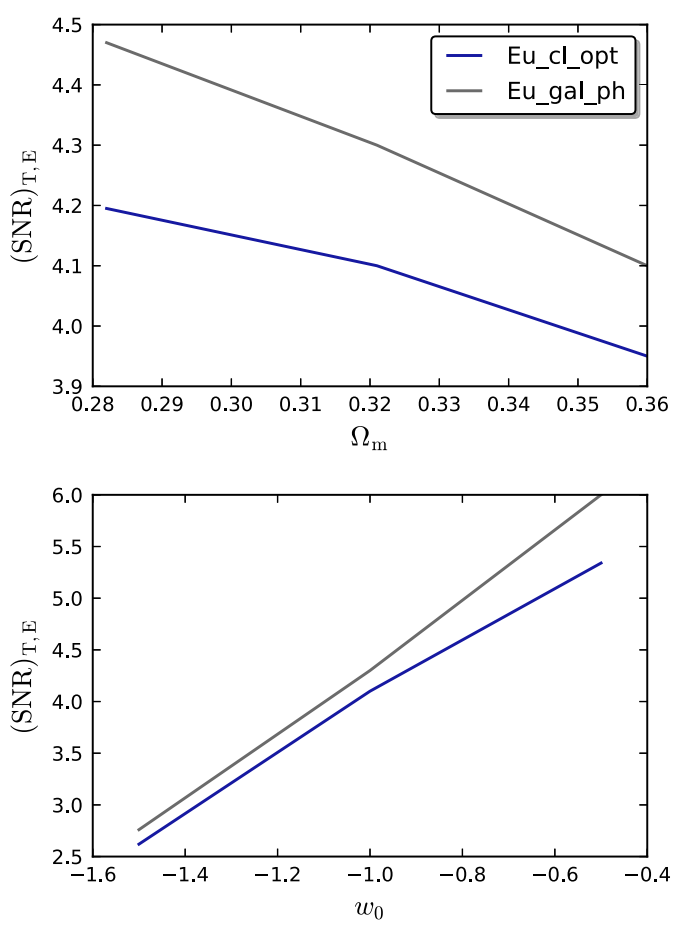

Figure 7. Top panel: dependence on $\Omega_{\mathrm{m}}$ of the SNR for the ISW detection with clusters (optimistic Euclid cluster survey, blue line) and with galaxies (photometric Euclid galaxy survey, grey line). Bottom panel: dependence on the dark energy equation of state $w_{0}$ of the SNR for the ISW detection. it offers the possibility of a more robust ISW detection when cosmological parameters are not kept fixed.

The combination of the two Euclid cluster surveys with Eu_gal_sp brings a little improvement of +0.2 in the SNR.

Finally, we find that for eROSITA the combination of cluster survey with the galaxy survey from Euclid will be more promising in order to raise the SNR thanks to the low-z coverage from $e$ ROSITA. We find a SNR of 3.2/4.1 by combining and cross-correlating eR_cl with Eu_gal_sp and Eu_gal_ph.

Also in this case the addition of the CMB polarization information increases the SNR by a further 18 per cent for all the combinations.

\section{COSMOLOGICAL FORECASTS WITH CMB-CLUSTER CROSS-CORRELATION}

In this section, we use the Fisher matrix technique (Tegmark, Taylor \& Heavens 1997) to compute the joint CMB and crosscorrelation forecast constraints on few extensions of the $\Lambda \mathrm{CDM}$ cosmological concordance model.

\subsection{Methodology}

We use the Fisher matrix derived from the Gaussian approximation of the full likelihood $\mathcal{L}$. By using the Gaussian hypothesis for the data $\boldsymbol{d}$, the likelihood function of the cosmological parameters $\theta$ reads as

$\mathcal{L}(\theta \mid \mathbf{d}) \propto \frac{1}{\sqrt{|\mathbf{C}(\theta)|}} \exp \left\{-\frac{1}{2}\left(\mathbf{d}^{\mathrm{obs}}\right)^{\dagger}[\mathbf{C}(\theta)]^{-1} \mathbf{d}^{\mathrm{obs}}\right\}$, 
where

$\mathbf{C}=\left(\begin{array}{lll}C_{\ell}^{\mathrm{TT}} & C_{\ell}^{\mathrm{TE}} & C_{\ell}^{\mathrm{TX}} \\ C_{\ell}^{\mathrm{TE}} & C_{\ell}^{\mathrm{EE}} & C_{\ell}^{\mathrm{EX}} \\ C_{\ell}^{\mathrm{TX}} & C_{\ell}^{\mathrm{EX}} & C_{\ell}^{\mathrm{XX}}\end{array}\right)$,

is the theoretical covariance matrix of the modelled data $\mathbf{d}=$ $\left\{a_{\ell m}^{\mathrm{T}}, a_{\ell m}^{\mathrm{E}}, a_{\ell m}^{\mathrm{X}}\right\}$ where $X=\{\mathrm{c}, \mathrm{g}\}$ and $|\mathbf{C}(\boldsymbol{\theta})|$ its determinant. From equation (25), we write the Fisher matrix in compact form as

$$
\begin{aligned}
\mathcal{F}_{\alpha \beta} & =\left\langle\frac{\partial^{2} \log \mathcal{L}}{\partial \theta_{\alpha} \partial \theta_{\beta}}\right\rangle, \\
& =\frac{1}{2} \operatorname{tr}\left[\frac{\partial \mathbf{C}}{\partial \theta_{\alpha}} \mathbf{C}^{-1} \frac{\partial \mathbf{C}}{\partial \theta_{\alpha}} \mathbf{C}^{-1}\right], \\
& =\sum_{\ell} \frac{(2 \ell+1) f_{\text {sky }}^{\mathrm{X}}}{2} \frac{\partial C_{\ell}^{\mathrm{AB}}}{\partial \theta_{\alpha}} \operatorname{Cov}^{-1}\left(C_{\ell}^{\mathrm{AB}}, C_{\ell}^{\mathrm{CD}}\right) \frac{\partial C_{\ell}^{\mathrm{CD}}}{\partial \theta_{\beta}},
\end{aligned}
$$

where $\bar{C}_{\ell}^{\mathrm{AB}}=C_{\ell}^{\mathrm{AB}}+\delta_{\mathrm{AB}} N_{\ell}^{\mathrm{A}}$ is the sum of the signal and the noise, with $\mathcal{N}_{\ell}^{\mathrm{TE}}, \mathcal{N}_{\ell}^{\mathrm{TX}}, \mathcal{N}_{\ell}^{\mathrm{EX}}=0$. For the temperature and polarization angular power spectra, here $\mathcal{N}_{\ell}^{\mathrm{T}, \mathrm{E}}=\sigma_{\mathrm{T}, \mathrm{E}} b_{\ell}^{-2}$ is the isotropic noise deconvolved with the instrument beam, $b_{\ell}^{2}$ is the beam window function, assumed Gaussian, with $b_{\ell}=\mathrm{e}^{-\ell(\ell+1) \theta_{\mathrm{FWHM}}^{2} / 16 \ln 2} ; \theta_{\mathrm{FWHM}}$ is the full width half-maximum (FWHM) of the beam in radians; $\sigma_{\mathrm{TT}}$ and $\sigma_{\mathrm{EE}}$ are the square of the detector noise level on a steradian patch for temperature and polarization, respectively. See the Appendix for the full structure of the covariance matrix Cov in equation (27).

As representative specifications for CMB we consider the Planck $143 \mathrm{GHz}$ channel full mission sensitivity and angular resolution as given in Adam et al. (2016) and used in Ballardini et al. (2016). These correspond to $\theta_{\mathrm{FWHM}}=7.3 \operatorname{arcmin}, \sigma_{\mathrm{T}}=33 \operatorname{arcmin} \mu \mathrm{K}$ and $\sigma_{\mathrm{E}}=70.2 \operatorname{arcmin} \mu \mathrm{K}$. For cluster and galaxy specifications, we use those listed in Table 1.

We consider as set of cosmological parameters in our forecast analysis the six standard $\Lambda \mathrm{CDM}$ parameters $\omega_{\mathrm{b}}, \omega_{\mathrm{c}}, H_{0}, \tau, n_{\mathrm{s}}$, and $\log \left(10^{10} A_{\mathrm{s}}\right)$, plus some extra parameters described in the following subsection. We also consider the bias as a nuisance parameter marginalizing over it.

In the case of clusters, in addition to the variation on all the cosmological parameters we consider the dependence on four nuisance parameters $B_{\mathrm{M}, 0}, \alpha, \sigma_{\log M, 0}^{2}, \beta$ introduced in Section 3.3, as sources of uncertainties on the cluster selection function, as in Sartoris et al. (2016), by marginalizing over them.

\subsection{Results}

In this paper, we restrict ourselves to forecast the joint CMB and ISW-cluster cross-correlation constraints on few relevant cosmological models as in Douspis et al. (2008). With this setting, the impact of ISW-cluster cross-correlation can be easily evaluated, whereas it would be hardly visible if we were considering the full combination of CMB and cluster cosmological information. As for the SNR in the previous section, we compare these results with those obtained by the joint CMB and ISW-galaxy cross-correlation expected from the Euclid spectroscopic and photometric surveys.

We first forecast the constraints on the redshift-independent parameter of state of dark energy $w_{0}$. Fig. 8 shows how the CMBcluster cross-correlation improves on the $\mathrm{CMB}$ results alone. Our results show that $\mathrm{CMB}$-cluster cross-correlation could lead to constraints similar to the one expected from the CMB-galaxy one, improving the constraints on $w_{0}$ obtained from $\mathrm{CMB}$ data alone by around 20-30 per cent.
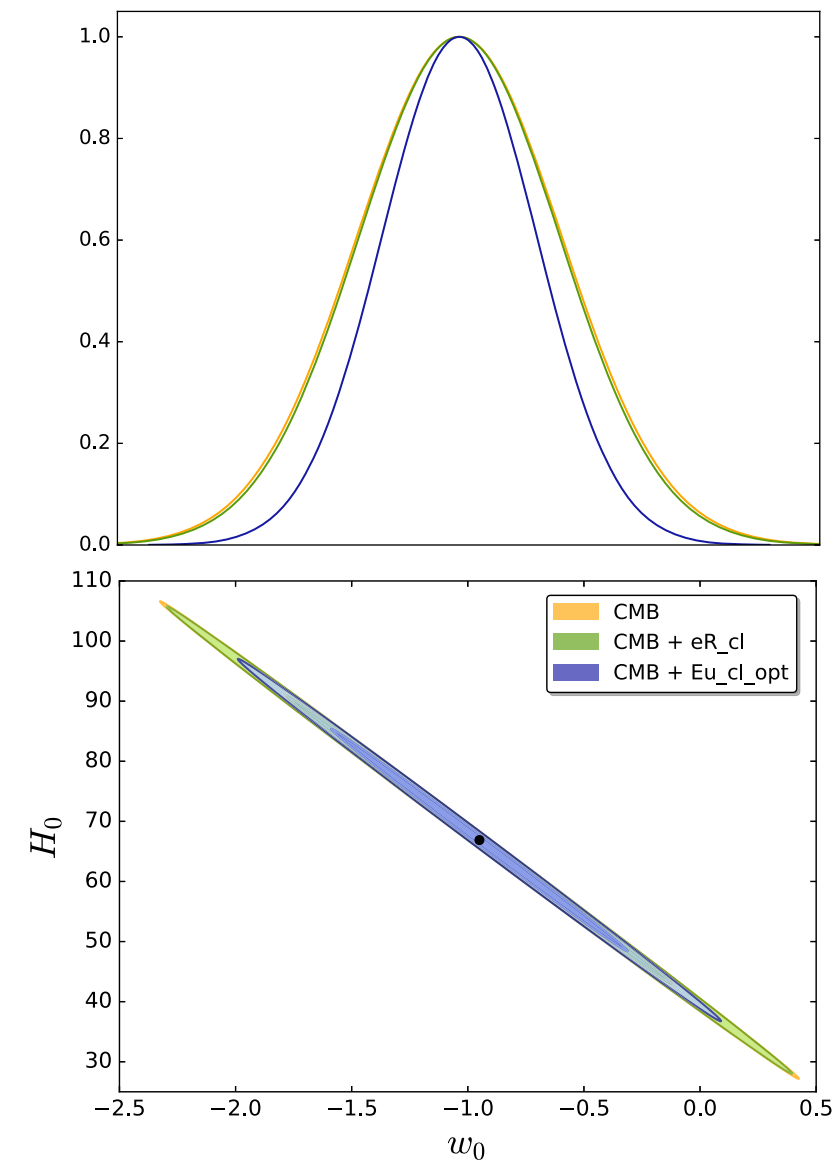

Figure 8. In the bottom panel, we show the joint constraints on $\left(w_{0}, H_{0}\right)$ at the 68 per cent and 95 per cent $\mathrm{CL}$. We show forecasts for the combination of the CMB and the CMB-LSS cross-correlation from eROSITA (green) and from the optimistic Euclid photometric cluster survey (blue). We show also the results for the CMB alone (yellow). In the top panel, we show the corresponding posterior distributions for $w_{0}$.

As second cosmological scenario, we show the constraints for a cosmology in which the parameter of state of dark energy is allowed to be redshift dependent (Chevallier \& Polarski 2001; Linder 2003):

$w(z)=w_{0}+\frac{z}{1+z} w_{a}$,

where $w_{0}$ is the present value of the equation of state and $w_{a}$ is its first derivative with respect to the scale parameter $a(t)$. Also in this case, we see from Fig. 9 that the addition of CMB-cluster crosscorrelations leads to a significant improvement for the dark energy parameters, with a similar performance between $e$ ROSITA and Euclid. The improvement with respect to $\mathrm{CMB}$-only constraints for the $w_{0}-w_{\mathrm{a}} \mathrm{CDM}$ cosmological model corresponds to 6 percent on $H_{0}, 4$ percent over $n_{\mathrm{s}}, 5$ percent on $w_{0}$, and 20 percent on $w_{a}$. The figure of merit (FoM) for the dark energy parameters (Albrecht et al. 2009) improves by a factor of 1.5 when the ISW-cluster cross-correlation is included. In Fig. 9, the difference between the orientation of the con tour of eROSITA and the one of Euclid in the $w_{0}-w_{\text {a }}$ parameters space is not only due to the different redshift range probed by the two surveys but also due to the larger sky coverage from RROSITA that helps in breaking the degeneracy between the two parameters.

We consider a cosmological model in which there is a suppression on large scales of the primodial curvature power spectrum (Contaldi 


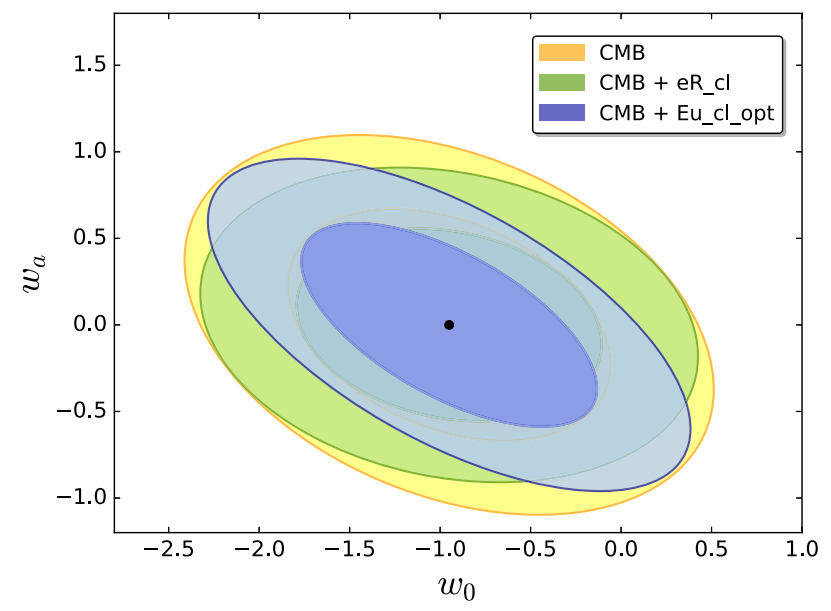

Figure 9. Joint constraints on $\left(w_{0}, w_{a}\right)$ at the 68 percent and 95 percent CL. We show forecasts for the combination of the CMB and the CMBLSS cross-correlation from eROSITA (green) and from the optimistic Euclid photometric cluster survey (blue). We show also the results for the CMB alone (yellow).

et al. 2003):

$\mathcal{P}_{\mathcal{R}}(k)=\mathcal{P}_{\mathcal{R}, 0}(k)\left\{1-\exp \left[-\left(\frac{k}{k_{\mathrm{c}}}\right)^{\lambda_{\mathrm{c}}}\right]\right\}$,

where $k_{\mathrm{c}}$ is the relevant scale of deviation from a power-law spectrum and $\lambda_{\mathrm{c}}$ adjusts the stiffness of the suppression. For this model, the addition of the CMB-cluster cross-correlation leads to a hardly visible improvement in the constraints on $\left(\lambda_{\mathrm{c}}, k_{\mathrm{c}}\right)$ with respect to the CMB constraints, since, even if on large scales, the suppression induces a change in the SW contribution rather than on the ISW one. Nevertheless, the ISW-LSS cross-correlation is useful to break degeneracies in the physical effects at play on the largest scales.

Note that in our calculations we have taken into account the dependence of both the halo mass and bias functions on the underlying cosmology through the variance of the fractional density fluctuation $\sigma^{2}(M, z)$. We consider this dependence when varying the angular power spectra $C_{\ell}^{\mathrm{cc}}$ and $C_{\ell}^{\mathrm{Tc}}$ around the fiducial cosmology in the calculation of the Fisher matrix (27). The effect of neglecting this dependence would lead to an increase in the uncertainties on the cosmological parameters of about 10 percent for the most significant parameters such as $w_{0}$, and $w_{\mathrm{a}}$.

As a semi-idealized case, we also consider the constraints on the $w_{0}-w_{\text {a }}$ plane by assuming perfect knowledge of the scaling relation between the true and the observed cluster mass, i.e. by fixing all the corresponding nuisance parameters: this semi-idealized case would cast the cluster case at the same footing of the galaxy one, in which no nuisance parameter is considered. In Fig. 10, we show the comparison of the constraints in the $w_{0}-w_{\text {a }}$ parameters space between the CMB-cluster cross-correlation from Eu_cl_opt, with and without perfect knowledge of the scaling relation, and the CMB-galaxy cross-correlation from Eu_gal_ph. Assuming a perfect knowledge of the nuisance parameters connected to the cluster selection function leads to an improvement in the constraints obtainable with the help the CMB-cluster cross-correlation. In particular, we found an improvement of 10 percent for $w_{0}$, of 20 percent and 40 percent for $w_{0}$ and $w_{a}$, of 5 percent for $k_{\mathrm{c}}$ and $\lambda_{\mathrm{c}}$.

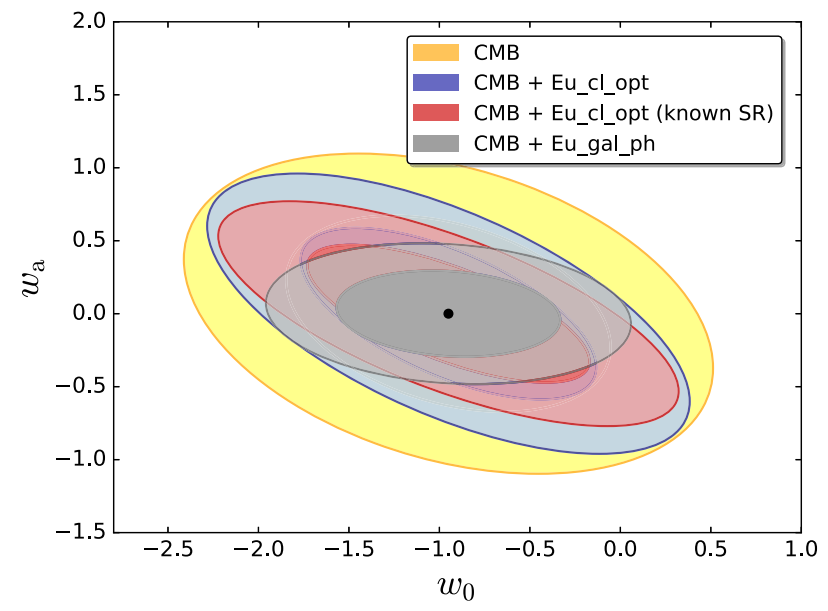

Figure 10. Joint constraints on $\left(w_{0}, w_{\mathrm{a}}\right)$ at the 68 per cent and 95 per cent CL. We show forecasts for the combination of the CMB and the CMBLSS cross-correlation from the optimistic Euclid photometric cluster survey with (red) and without (blue) assuming a perfect knowledge of the scaling relation, and from the photometric Euclid galaxy survey (grey). We also show the results for the CMB alone for comparison (yellow).

\section{CONCLUSIONS}

Cosmology with clusters is a rapidly evolving field, thanks to improvements in observations. Current catalogues are already sufficient to obtain an accurate measurement of the baryon acoustic oscillation with galaxy clusters as from the Sloan Digital Sky Survey leading to a distance-redshift relation in full agreement with Planck observations and with uncertainties similar to the one obtained by galaxy surveys (Veropalumbo et al. 2014, 2016). In the perspective of future surveys in several wavelengths which will provide catalogues with a higher number of clusters over a wider redshift range, we have studied the capabilities to detect the ISW effect by a CMB-cluster cross-correlation. Note that this ISW-cluster cross-correlation is not internal to CMB as the previously studied ISW-tSZ cross-correlation (Taburet et al. 2011). In this perspective, it will be essential to control the SZ cluster residuals in CMB maps that could bias the cross-correlation measurements between clusters and CMB (Chen, Remazeilles \& Dickinson 2018).

As two representative cases, we have considered the specifications of the cluster catalogues as expected from two coming space missions, i.e. eROSITA (Predehl et al. 2010) and Euclid (Laureijs et al. 2011). We have found that the CMB-cluster cross-correlation could be used for a statistically significant detection of the ISW effect. As two general remarks, we have found that (1) the SNR of the CMB-cluster cross-correlation benefits of the large cluster bias that balances the larger shot-noise in cluster catalogues compared to galaxy surveys; (2) the dependence of the cluster bias on cosmology needs to be taken into account to extract the full information from the cross-correlation with CMB. Whereas for $e$ ROSITA the cross-correlation with $\mathrm{CMB}$ would be an added value for the legacy of its cluster catalogue, for Euclid it would constitute a sort of coarse graining of the CMB galaxy cross-correlation and a key verification of the structure formation process. Nevertheless, the CMB-cluster would be another valuable cross-correlation within the Euclid probes and would add useful information on the bias for the cluster catalogue. The cross-correlation studied here would be of interest for other surveys as well, as LSST (Abell et al. 2009) and SKA (Maartens et al. 2015). 


\section{ACKNOWLEDGEMENTS}

We thank Nabila Aghanim and Carlo Baccigalupi for useful comments to the paper. We acknowledge financial contribution from the agreement ASI n.I/023/12/0 'Attività relative alla fase B2/C per la missione Euclid'. The support by the 'ASI/INAF Agreement 2014-024-R.0 for the Planck LFI Activity of Phase E2' is also acknowledged. MB, DP, FF, and LM acknowledge the support from the grant MIUR PRIN 2015 'Cosmology and Fundamental Physics: illuminating the Dark Universe with Euclid'. MB was supported by the South African Radio Astronomy Observatory, which is a facility of the National Research Foundation, an agency of the Department of Science and Technology and the Claude Leon Foundation. BS acknowledges financial support from the University of Trieste through the program 'Finanziamento di Ateneo per progetti di ricerca scientifica - FRA 2015', a grant from 'Consorzio per la Fisica - Trieste' and from the PRIN 2015W7KAWC project, funded by the Italian Minister for University and Research.

\section{REFERENCES}

Adam R.Planck, et al., Planck, 2016, A\&A, 594, A1

Afshordi N., 2004, Phys. Rev. D, 70, 083536

Afshordi N., Loh Y.-S., Strauss M. A., 2004, Phys. Rev. D, 69, 083524

Albrecht A. et al., 2009, preprint (arXiv:0901.0721)

Amendola L. et al., 2013, Living Rev. Rel., 16, 6

Ballardini M., Finelli F., Fedeli C., Moscardini L., 2016, JCAP, 1610, 041

Bianchini F., Renzi A., Marinucci D., 2016, JCAP, 1611, 050

Boughn S., Crittenden R., 2004, Nature, 427, 45

Chen T., Remazeilles M., Dickinson C., 2018, MNRAS, 479, 4239

Chevallier M., Polarski D., 2001, Int. J. Mod. Phys. D, 10, 213

Contaldi C. R., Peloso M., Kofman L., Linde A. D., 2003, JCAP, 0307, 002

Cooray A., 2002, Phys. Rev. D, 65, 103510

Cooray A., Melchiorri A., 2006, JCAP, 0601, 018

Crittenden R. G., Turok N., 1996, Phys. Rev. Lett., 76, 575

DESI Collaboration, et al., 2013, preprint (arXiv:1308.0847)

DESI Collaboration, et al., 2016a, preprint (arXiv:1611.00036)

DESI Collaboration, et al., 2016b, preprint (arXiv:1611.00037)

Douspis M., Castro P. G., Caprini C., Aghanim N., 2008, A\&A, 485, 395

Dupe F. X., Rassat A., Starck J. L., Fadili M. J., 2011, A\&A, 534, A51

Ferraro S., Sherwin B. D., Spergel D. N., 2015, Phys. Rev. D, 91, 083533

Fosalba P., Gaztanaga E., 2004, MNRAS, 350, L37

Frommert M., Enßlin T. A., 2009, MNRAS, 395, 1837

Giannantonio T., Crittenden R., Nichol R., Ross A. J., 2012, MNRAS, 426, 2581

Giannantonio T., Scranton R., Crittenden R. G., Nichol R. C., Boughn S. P., Myers A. D., Richards G. T., 2008, Phys. Rev. D, 77, 123520

Granett B. R., Neyrinck M. C., Szapudi I., 2008, ApJ, 683, L99

Hernandez-Monteagudo C., 2010, A\&A, 520, A101

Ho S., Hirata C., Padmanabhan N., Seljak U., Bahcall N., 2008, Phys. Rev. D, 78, 043519

Ilic S., Douspis M., Langer M., Penin A., Lagache G., 2011, MNRAS, 416, 2688

Kofman L., Starobinsky A. A., 1985, Sov. Astron. Lett., 11, 271

Laureijs R.Euclid, et al., Euclid, 2011, preprint (arXiv:1110.3193)

Lima M., Hu W., 2005, Phys. Rev. D, 72, 043006

Linder E. V., 2003, Phys. Rev. D, 68, 083504

LSST Science, LSST Project, et al., 2009, preprint (arXiv:0912.0201)

Maartens R., Abdalla F. B., Jarvis M., Santos M. G., 2015, PoS, AASKA14, 016

Mantz A. B., Allen S. W., Morris R. G., Schmidt R. W., von der Linden A., Urban O., 2015, MNRAS, 449, 199

Manzotti A., Dodelson S., 2014, Phys. Rev. D, 90, 123009

McEwen J. D., Vielva P., Hobson M. P., Martinez-Gonzalez E., Lasenby A. N., 2007, MNRAS, 376, 1211

Nolta M. R. et al., 2004, ApJ, 608, 10
Papai P., Szapudi I., Granett B. R., 2011, ApJ, 732, 27

Pillepich A., Porciani C., Reiprich T. H., 2012, MNRAS, 422, 44

Planck Collaboration XIX, 2014, A\&A, 571, A19

Planck Collaboration XLVI, , 2016, A\&A, 596, A107

Planck Collaboration et al., 2016, A\&A, 594, A21

Pourtsidou A., Bacon D., Crittenden R., 2017, MNRAS, 470, 4251

Pozzetti L. et al., 2016, A\&A, 590, A3

Predehl P. et al., 2010, Proc. SPIE , 7732, 77320U

Raccanelli A., Kovetz E., Dai L., Kamionkowski M., 2016, Phys. Rev. D, 93, 083512

Raccanelli A. et al., 2015, JCAP, 1501, 042

Rozo E. et al., 2010, ApJ, 708, 645

Sachs R. K., Wolfe A. M., 1967, ApJ, 147, 73

Sartoris B., Borgani S., Fedeli C., Matarrese S., Moscardini L., Rosati P., Weller J., 2010, MNRAS, 407, 2339

Sartoris B. et al., 2016, MNRAS, 459, 1764

Schiavon F., Finelli F., Gruppuso A., Marcos-Caballero A., Vielva P., Crittenden R. G., Barreiro R. B., Martinez-Gonzalez E., 2012, MNRAS, 427, 3044

Shajib A. J., Wright E. L., 2016, ApJ, 827, 116

Stölzner B., Cuoco A., Lesgourgues J., Bilicki M., 2017

Taburet N., Hernandez-Monteagudo C., Aghanim N., Douspis M., Sunyaev R. A., 2011, MNRAS, 418, 2207

Tegmark M., Taylor A., Heavens A., 1997, ApJ, 480, 22

Tinker J. L., Kravtsov A. V., Klypin A., Abazajian K., Warren M. S., Yepes G., Gottlober S., Holz D. E., 2008, ApJ, 688, 709

Tinker J. L., Robertson B. E., Kravtsov A. V., Klypin A., Warren M. S., Yepes G., Gottlober S., 2010, ApJ, 724, 878

Veropalumbo A., Marulli F., Moscardini L., Moresco M., Cimatti A., 2014, MNRAS, 442, 3275

Veropalumbo A., Marulli F., Moscardini L., Moresco M., Cimatti A., 2016, MNRAS, 458, 1909

Vielva P., Martinez-Gonzalez E., Tucci M., 2006, MNRAS, 365, 891

Zaldarriaga M., 1997, Phys. Rev. D, 55, 1822

\section{APPENDIX: THE COVARIANCE IN THE FISHER APPROACH}

Since we are interested in the effect coming from the crosscorrelation between the CMB and LSS surveys alone, we do not consider information coming from the LSS tracer angular power spectrum, i.e. we use $C_{\ell}^{\mathrm{XX}}$ just for the covariance. The non-vanishing elements of the symmetric angular power-spectrum covariance matrix $\operatorname{Cov}_{\ell}$ at the $\ell$ th multipole are

$\operatorname{Cov}\left(C_{\ell}^{\mathrm{AB}}, C_{\ell}^{\mathrm{CD}}\right)=\bar{C}_{\ell}^{\mathrm{AC}} \bar{C}_{\ell}^{\mathrm{BD}}+\bar{C}_{\ell}^{\mathrm{AD}} \bar{C}_{\ell}^{\mathrm{BC}}$

The exact structure of $\mathrm{Cov}_{\ell}^{-1}$ is more useful compared to its inverse, since it allows to derive all the reduced cases for its inverse when some cross-correlation terms go to zero:

$$
\begin{aligned}
\operatorname{Cov}_{\ell}^{-1}= & \frac{1}{(|\mathbf{C}(\theta)|)^{2}} \\
& \times\left(\begin{array}{llllll}
\Sigma_{\text {TTTT }} & \Sigma_{\text {TTEE }} & \Sigma_{\text {TTTE }} & \Sigma_{\text {TTXX }} & \Sigma_{\text {TTTX }} & \Sigma_{\text {TTEX }} \\
\Sigma_{\text {TTEE }} & \Sigma_{\text {EEEE }} & \Sigma_{\text {EETE }} & \Sigma_{\text {EEXX }} & \Sigma_{\text {EETX }} & \Sigma_{\text {EEEX }} \\
\Sigma_{\text {TTTE }} & \Sigma_{\text {EETE }} & \Sigma_{\text {TETE }} & \Sigma_{\text {TEXX }} & \Sigma_{\text {TETX }} & \Sigma_{\text {TEEX }} \\
\Sigma_{\text {TTXX }} & \Sigma_{\text {EEXX }} & \Sigma_{\text {TEXX }} & \Sigma_{\text {XXXX }} & \Sigma_{\text {XXTX }} & \Sigma_{\text {XXEX }} \\
\Sigma_{\text {TTTX }} & \Sigma_{\text {EETX }} & \Sigma_{\text {TETX }} & \Sigma_{\text {XXTX }} & \Sigma_{\text {TXTX }} & \Sigma_{\text {TXEX }} \\
\Sigma_{\text {TTEX }} & \Sigma_{\text {EEXX }} & \Sigma_{\text {TEXX }} & \Sigma_{\text {XXEX }} & \Sigma_{\text {TXEX }} & \Sigma_{\text {EXEX }}
\end{array}\right),
\end{aligned}
$$

where the autocorrelation are

$$
\begin{aligned}
& \Sigma_{\mathrm{TTTT}}=\left[\left(\bar{C}_{\ell}^{\mathrm{EX}}\right)^{2}-\bar{C}_{\ell}^{\mathrm{EE}} \bar{C}_{\ell}^{\mathrm{XX}}\right]^{2}, \\
& \Sigma_{\mathrm{EEEE}}=\left[\left(\bar{C}_{\ell}^{\mathrm{TX}}\right)^{2}-\bar{C}_{\ell}^{\mathrm{TT}} \bar{C}_{\ell}^{\mathrm{XX}}\right]^{2},
\end{aligned}
$$




$$
\begin{aligned}
\Sigma_{\mathrm{TETE}}= & 2\left\{2\left(\bar{C}_{\ell}^{\mathrm{TX}}\right)^{2}\left(\bar{C}_{\ell}^{\mathrm{EX}}\right)^{2}\right. \\
& +\left(\bar{C}_{\ell}^{\mathrm{XX}}\right)^{2}\left[\left(\bar{C}_{\ell}^{\mathrm{TE}}\right)^{2}-\bar{C}_{\ell}^{\mathrm{TT}} \bar{C}_{\ell}^{\mathrm{EE}}\right] \\
& -\bar{C}_{\ell}^{\mathrm{XX}}\left[2 \bar{C}_{\ell}^{\mathrm{TE}} \bar{C}_{\ell}^{\mathrm{TX}} \bar{C}_{\ell}^{\mathrm{EX}}+\bar{C}_{\ell}^{\mathrm{TT}}\left(\bar{C}_{\ell}^{\mathrm{EX}}\right)^{2}\right. \\
& \left.\left.+\bar{C}_{\ell}^{\mathrm{EE}}\left(\bar{C}_{\ell}^{\mathrm{TX}}\right)^{2}\right]\right\},
\end{aligned}
$$$$
\Sigma_{\mathrm{XXXX}}=\left[\left(\bar{C}_{\ell}^{\mathrm{TE}}\right)^{2}-\bar{C}_{\ell}^{\mathrm{TT}} \bar{C}_{\ell}^{\mathrm{EE}}\right]^{2},
$$$$
\Sigma_{\mathrm{TXTX}}=2\left\{-2 \bar{C}_{\ell}^{\mathrm{EE}} \bar{C}_{\ell}^{\mathrm{TE}} \bar{C}_{\ell}^{\mathrm{TX}} \bar{C}_{\ell}^{\mathrm{EX}}\right.
$$

$$
\begin{aligned}
& +\left(\bar{C}_{\ell}^{\mathrm{EX}}\right)^{2}\left[2\left(\bar{C}_{\ell}^{\mathrm{TE}}\right)^{2}-\bar{C}_{\ell}^{\mathrm{TT}} \bar{C}_{\ell}^{\mathrm{EE}}\right] \\
& +\bar{C}_{\ell}^{\mathrm{EE}}\left[\bar{C}_{\ell}^{\mathrm{TT}} \bar{C}_{\ell}^{\mathrm{EE}} \bar{C}_{\ell}^{\mathrm{XX}}+\bar{C}_{\ell}^{\mathrm{EE}}\left(\bar{C}_{\ell}^{\mathrm{TX}}\right)^{2}\right. \\
& \left.\left.-\bar{C}_{\ell}^{\mathrm{XX}}\left(\bar{C}_{\ell}^{\mathrm{TE}}\right)^{2}\right]\right\},
\end{aligned}
$$

$$
\begin{aligned}
\Sigma_{\mathrm{EXEX}}= & 2\left\{2\left(\bar{C}_{\ell}^{\mathrm{TE}}\right)^{2}\left(\bar{C}_{\ell}^{\mathrm{TX}}\right)^{2}-\bar{C}_{\ell}^{\mathrm{XX}}\left(\bar{C}_{\ell}^{\mathrm{TE}}\right)^{2}\right. \\
& +\left(\bar{C}_{\ell}^{\mathrm{TT}}\right)^{2}\left[\left(\bar{C}_{\ell}^{\mathrm{EX}}\right)^{2}+\bar{C}_{\ell}^{\mathrm{EE}} \bar{C}_{\ell}^{\mathrm{XX}}\right] \\
& \left.-\bar{C}_{\ell}^{\mathrm{TX}}\left(2 \bar{C}_{\ell}^{\mathrm{TE}} \bar{C}_{\ell}^{\mathrm{EX}}+\bar{C}_{\ell}^{\mathrm{EE}} \bar{C}_{\ell}^{\mathrm{TX}}\right)\right\},
\end{aligned}
$$

\section{and the cross-correlation are}

$$
\begin{aligned}
& \Sigma_{\mathrm{TTEE}}=\left[\bar{C}_{\ell}^{\mathrm{XX}} \bar{C}_{\ell}^{\mathrm{TE}}-\bar{C}_{\ell}^{\mathrm{EX}} \bar{C}_{\ell}^{\mathrm{TX}}\right]^{2}, \\
& \Sigma_{\mathrm{TTTE}}=2\left[\left(\bar{C}_{\ell}^{\mathrm{EX}}\right)^{2}-\bar{C}_{\ell}^{\mathrm{EE}} \bar{C}_{\ell}^{\mathrm{XX}}\right]\left(\bar{C}_{\ell}^{\mathrm{TE}} \bar{C}_{\ell}^{\mathrm{XX}}-\bar{C}_{\ell}^{\mathrm{TX}} \bar{C}_{\ell}^{\mathrm{EX}}\right), \\
& \Sigma_{\mathrm{TTXX}}=\left(\bar{C}_{\ell}^{\mathrm{TE}} \bar{C}_{\ell}^{\mathrm{EX}}-\bar{C}_{\ell}^{\mathrm{EE}} \bar{C}_{\ell}^{\mathrm{TX}}\right)^{2}, \\
& \Sigma_{\mathrm{TTTX}}=2\left[\left(\bar{C}_{\ell}^{\mathrm{EX}}\right)^{2}-\bar{C}_{\ell}^{\mathrm{EE}} \bar{C}_{\ell}^{\mathrm{XX}}\right]\left(\bar{C}_{\ell}^{\mathrm{EE}} \bar{C}_{\ell}^{\mathrm{TX}}-\bar{C}_{\ell}^{\mathrm{TE}} \bar{C}_{\ell}^{\mathrm{EX}}\right), \\
& \Sigma_{\mathrm{TTEX}}=2\left(\bar{C}_{\ell}^{\mathrm{TE}} \bar{C}_{\ell}^{\mathrm{EX}}-\bar{C}_{\ell}^{\mathrm{EE}} \bar{C}_{\ell}^{\mathrm{TX}}\right)\left(\bar{C}_{\ell}^{\mathrm{TX}} \bar{C}_{\ell}^{\mathrm{EX}}-\bar{C}_{\ell}^{\mathrm{TE}} \bar{C}_{\ell}^{\mathrm{XX}}\right),
\end{aligned}
$$

$\Sigma_{\mathrm{EETE}}=2\left[\left(-\bar{C}_{\ell}^{\mathrm{TX}}\right)^{2}+\bar{C}_{\ell}^{\mathrm{TT}} \bar{C}_{\ell}^{\mathrm{XX}}\right]\left(\bar{C}_{\ell}^{\mathrm{TX}} \bar{C}_{\ell}^{\mathrm{EX}}-\bar{C}_{\ell}^{\mathrm{TE}} \bar{C}_{\ell}^{\mathrm{XX}}\right),(\mathrm{A} 14)$

$$
\begin{aligned}
& \Sigma_{\mathrm{EEXX}}=\left(\bar{C}_{\ell}^{\mathrm{TE}} \bar{C}_{\ell}^{\mathrm{TX}}-\bar{C}_{\ell}^{\mathrm{TT}} \bar{C}_{\ell}^{\mathrm{EX}}\right)^{2}, \\
& \Sigma_{\mathrm{EETX}}=2\left(\bar{C}_{\ell}^{\mathrm{TE}} \bar{C}_{\ell}^{\mathrm{XX}}-\bar{C}_{\ell}^{\mathrm{TX}} \overline{\boldsymbol{C}}_{\ell}^{\mathrm{EX}}\right)\left(\bar{C}_{\ell}^{\mathrm{TT}} \bar{C}_{\ell}^{\mathrm{EX}}-\bar{C}_{\ell}^{\mathrm{TE}} \bar{C}_{\ell}^{\mathrm{TX}}\right),
\end{aligned}
$$

$$
\begin{aligned}
\Sigma_{\mathrm{EEEX}}= & 2\left[\left(\bar{C}_{\ell}^{\mathrm{TX}}\right)^{2}-\bar{C}_{\ell}^{\mathrm{TT}} \bar{C}_{\ell}^{\mathrm{XX}}\right]\left(\bar{C}_{\ell}^{\mathrm{TT}} \bar{C}_{\ell}^{\mathrm{EX}}-\bar{C}_{\ell}^{\mathrm{TE}} \bar{C}_{\ell}^{\mathrm{TX}}\right), \\
\Sigma_{\mathrm{TEXX}}= & 2\left(\bar{C}_{\ell}^{\mathrm{EE}} \bar{C}_{\ell}^{\mathrm{TX}}-\bar{C}_{\ell}^{\mathrm{TE}} \bar{C}_{\ell}^{\mathrm{EX}}\right)\left(\bar{C}_{\ell}^{\mathrm{TT}} \bar{C}_{\ell}^{\mathrm{EX}}-\bar{C}_{\ell}^{\mathrm{TE}} \bar{C}_{\ell}^{\mathrm{TX}}\right), \\
\Sigma_{\mathrm{TETX}}= & -2\left\{-2 \bar{C}_{\ell}^{\mathrm{EE}} \bar{C}_{\ell}^{\mathrm{TE}} \bar{C}_{\ell}^{\mathrm{XX}} \bar{C}_{\ell}^{\mathrm{TX}}-\bar{C}_{\ell}^{\mathrm{TT}}\left(\bar{C}_{\ell}^{\mathrm{EX}}\right)^{3}\right. \\
& +\bar{C}_{\ell}^{\mathrm{EX}}\left[\left(\bar{C}_{\ell}^{\mathrm{TX}}\right)^{2} \bar{C}_{\ell}^{\mathrm{EE}}\right. \\
& \left.\left.+\bar{C}_{\ell}^{\mathrm{XX}}\left[\left(\bar{C}_{\ell}^{\mathrm{TE}}\right)^{2}+\bar{C}_{\ell}^{\mathrm{TT}} \bar{C}_{\ell}^{\mathrm{EE}}\right]\right]\right\},
\end{aligned}
$$

$$
\begin{aligned}
\Sigma_{\mathrm{TEEX}}= & -2\left\{\left(\bar{C}_{\ell}^{\mathrm{EX}}\right)^{2} \bar{C}_{\ell}^{\mathrm{TT}} \bar{C}_{\ell}^{\mathrm{TX}}-\bar{C}_{\ell}^{\mathrm{EE}}\left(\bar{C}_{\ell}^{\mathrm{TX}}\right)^{3}\right. \\
& +\bar{C}_{\ell}^{\mathrm{XX}}\left[\left(\bar{C}_{\ell}^{\mathrm{TE}}\right)^{2} \bar{C}_{\ell}^{\mathrm{TX}}-2 \bar{C}_{\ell}^{\mathrm{TT}} \bar{C}_{\ell}^{\mathrm{TE}} \bar{C}_{\ell}^{\mathrm{EX}}\right. \\
& \left.\left.+\bar{C}_{\ell}^{\mathrm{TT}} \bar{C}_{\ell}^{\mathrm{EE}} \bar{C}_{\ell}^{\mathrm{TX}}\right]\right\},
\end{aligned}
$$

$$
\Sigma_{\mathrm{XXTX}}=2\left[\left(\bar{C}_{\ell}^{\mathrm{TE}}\right)^{2}-\bar{C}_{\ell}^{\mathrm{EE}} \bar{C}_{\ell}^{\mathrm{TT}}\right]\left(\bar{C}_{\ell}^{\mathrm{EE}} \bar{C}_{\ell}^{\mathrm{TX}}-\bar{C}_{\ell}^{\mathrm{TE}} \bar{C}_{\ell}^{\mathrm{EX}}\right),
$$

$$
\Sigma_{\mathrm{XXEX}}=2\left[\left(\bar{C}_{\ell}^{\mathrm{TE}}\right)^{2}-\bar{C}_{\ell}^{\mathrm{EE}} \bar{C}_{\ell}^{\mathrm{TT}}\right]\left(\bar{C}_{\ell}^{\mathrm{TT}} \bar{C}_{\ell}^{\mathrm{EX}}-\bar{C}_{\ell}^{\mathrm{TX}} \bar{C}_{\ell}^{\mathrm{TE}}\right),
$$

$$
\begin{aligned}
\Sigma_{\mathrm{TXEX}}= & -2\left\{\left(\bar{C}_{\ell}^{\mathrm{EX}}\right)^{2} \bar{C}_{\ell}^{\mathrm{TT}} \bar{C}_{\ell}^{\mathrm{TE}}\right. \\
& +\bar{C}_{\ell}^{\mathrm{EE}} \bar{C}_{\ell}^{\mathrm{TX}}\left(\bar{C}_{\ell}^{\mathrm{TE}} \bar{C}_{\ell}^{\mathrm{TX}}-2 \bar{C}_{\ell}^{\mathrm{TT}} \bar{C}_{\ell}^{\mathrm{EX}}\right) \\
& \left.+\bar{C}_{\ell}^{\mathrm{XX}}\left[\bar{C}_{\ell}^{\mathrm{TT}} \bar{C}_{\ell}^{\mathrm{EE}} \bar{C}_{\ell}^{\mathrm{TE}}-\left(\bar{C}_{\ell}^{\mathrm{TE}}\right)^{3}\right]\right\} .
\end{aligned}
$$

This paper has been typeset from a $\mathrm{T}_{\mathrm{E}} \mathrm{X} / \mathrm{LT} \mathrm{E} \mathrm{X}$ file prepared by the author. 